\title{
Inefficiency of equilibria in digital mechanisms with continuous valuations.
}

Citation for published version (APA):

Grigorieva, E., Herings, P. J. J., Müller, R. J., \& Vermeulen, A. J. (2011). Inefficiency of equilibria in digital mechanisms with continuous valuations. Journal of Mathematical Economics, 47, 541-544. https://doi.org/10.1016/j.jmateco.2011.06.002

Document status and date:

Published: 01/01/2011

DOI:

10.1016/j.jmateco.2011.06.002

Document Version:

Publisher's PDF, also known as Version of record

Document license:

Taverne

Please check the document version of this publication:

- A submitted manuscript is the version of the article upon submission and before peer-review. There can be important differences between the submitted version and the official published version of record.

People interested in the research are advised to contact the author for the final version of the publication, or visit the DOI to the publisher's website.

- The final author version and the galley proof are versions of the publication after peer review.

- The final published version features the final layout of the paper including the volume, issue and page numbers.

Link to publication

\footnotetext{
General rights rights.

- You may freely distribute the URL identifying the publication in the public portal. please follow below link for the End User Agreement:

www.umlib.nl/taverne-license

Take down policy

If you believe that this document breaches copyright please contact us at:

repository@maastrichtuniversity.nl

providing details and we will investigate your claim.
}

Copyright and moral rights for the publications made accessible in the public portal are retained by the authors and/or other copyright owners and it is a condition of accessing publications that users recognise and abide by the legal requirements associated with these

- Users may download and print one copy of any publication from the public portal for the purpose of private study or research.

- You may not further distribute the material or use it for any profit-making activity or commercial gain

If the publication is distributed under the terms of Article $25 \mathrm{fa}$ of the Dutch Copyright Act, indicated by the "Taverne" license above, 


\title{
Inefficiency of equilibria in digital mechanisms with continuous valuations
}

\author{
Elena Grigorieva ${ }^{\mathrm{a}}$, P. Jean-Jacques Herings ${ }^{\mathrm{b}, *}$, Rudolf Müller ${ }^{\mathrm{a}}$, Dries Vermeulen ${ }^{\mathrm{a}}$ \\ ${ }^{a}$ Department of Quantitative Economics, Maastricht University, P.O. Box 616, 6200 MD Maastricht, The Netherlands \\ ${ }^{\mathrm{b}}$ Department of Economics, Maastricht University, P.O. Box 616, 6200 MD Maastricht, The Netherlands
}

\section{A R T I C L E I N F O}

\section{Article history:}

Received 14 January 2010

Received in revised form

10 May 2011

Accepted 14 June 2011

Available online 8 July 2011

\section{Keywords:}

Mechanisms

Ex post equilibrium

Efficiency

\begin{abstract}
A B S T R A C T
A digital mechanism is defined as an iterative procedure in which bidders select an action, from a finite set, in each iteration. When bidders have continuous valuations and make strategic reports, we show that any ex post implementation of the Vickrey choice rule via such a mechanism needs infinitely many iterations for almost all realizations of the bidders' valuations. Thus, when valuations are drawn from a continuous probability distribution, the Vickrey choice rule can only be used at the expense of a running time that is infinite with probability one. This infeasibility result even holds in the case of two bidders and the Vickrey choice rule only being required to be established with probability one. Establishing the efficient allocation when the $n$ bidders' report truthfully contrasts starkly to the previous setting: a bisection procedure has a finite running time almost always, and an expected number of reports are equal to $2 n$. Using a Groves payment scheme rather than Vickrey's second price payment scheme somewhat mitigates the problem. We provide an example mechanism with a Groves payment scheme, in which the running time of the mechanism in equilibrium is finite with probability $\frac{1}{2}$.
\end{abstract}

(C) 2011 Elsevier B.V. All rights reserved.

\section{Introduction}

This paper concerns the sale of a single indivisible object to $n$ buyers by means of an auction, where buyer $i$ has a private valuation $v_{i}$ for the object. ${ }^{1}$ In this setting the Vickrey auction, introduced by Vickrey (1961), collects sealed bids, allocates the item to a bidder with the highest bid, and sets the price equal to the second-highest bid. In equilibrium, the Vickrey auction solves the allocation problem in dominant strategies and awards the object to a bidder with the highest valuation, thus ensuring allocative efficiency.

Despite these theoretical advantages, the Vickrey auction relies on exact communication of valuations between bidders and auctioneer. This is not feasible when bidders' valuations are continuous and communication takes place via a digitized communication channel. Irrespective of the language used to communicate valuations, the number of expressible elements in any digitized language is at most countable. In such a setting, it is necessary to rely on protocols that use finite-length messages, which are sent in discrete rounds to the auctioneer. Such protocols

\footnotetext{
* Corresponding author.

E-mail addresses: e.grigorieva@ke.unimaas.nl (E. Grigorieva), p.herings@maastrichtuniversity.nl, P.Herings@algec.unimaas.nl (P.J.J. Herings), r.muller@maastrichtuniversity.nl (R. Müller), d.vermeulen@maastrichtuniversity.nl (D. Vermeulen).

1 We assume quasi-linear utilities.
}

induce extensive form games. To gain flexibility, we allow for infinite histories in these games. ${ }^{2}$ Such protocols are referred to as digital mechanisms. ${ }^{3}$

All digital mechanisms for the sale of a single indivisible object, which have been proposed in the literature, may allocate the object inefficiently when valuations are continuous. For example, the English auction with a fixed bid increment is not efficient in this setting. Indeed the same is true for any English auction with discrete bid levels, as reported by David et al. (2005). In the same setting, Grigorieva et al. (2009) show that $c$-bisection auctions allocate inefficiently in equilibrium. Rothkopf and Harstad (1994) study a model with continuous valuations where bidders can only bid on a finite number of bid levels. They analyze how the choice of bid levels influences inefficiency and revenue under the assumption of straightforward bidding by the bidders.

On the other hand, the task of finding the efficient allocation of the object can easily be achieved by a digital mechanism if bidders are obedient. A bisection protocol, such as that proposed

\footnotetext{
2 As we focus on impossibility results, allowing for infinite histories only strengthens our results.

3 Another possible notion for such game forms would be communication protocol. However, Fadel and Segal (2009) use communication protocol for the combination of an iterative procedure and a particular bidding strategy, and the term binary dynamic mechanism for the extensive form game induced by their notion of communication protocol. As we like to keep mechanism and strategies separate, but stay consistent with their notation, we opt to not make use of either of the terms communication protocol or binary dynamic mechanism.
} 
in Arrow et al. (1981), can identify a bidder with maximum value for almost all combinations of valuations. The discrepancy between the setting with obedient bidders and the setting with strategic bidders has been called the cost of selfishness in Fadel and Segal (2009). They study a model with a finite set of valuations, and compare the amount of communication needed to enforce truthful reporting when agents report strategically versus when agents report obediently. They show that the average number of messages, in any efficient ex post equilibrium of a digital mechanism, tends to infinity as the set of possible valuations becomes large. The same comparison for the bisection protocol also shows that the cost of selfishness goes to infinity as the set of valuations becomes large. Arbitrarily close approximate efficiency is however achievable in equilibrium using digital mechanisms. Grigorieva et al. (2009) show that, for any $c>0$, the probability of inefficient allocation in equilibrium by the $c$-bisection auction is less than $c$, independent of the number of participants in the auction. Furthermore, the $c$-bisection auction ends after finitely many rounds, for all realizations of the valuations.

In this paper, we show that the result of Grigorieva et al. (2009) is the strongest result that can be achieved. When valuations are continuous, any equilibrium in a digital mechanism that implements the Vickrey choice rule requires an infinite number of binary messages, for almost all realizations of the valuations. This is a stronger result than that which can be concluded by taking limits in the discrete model of Fadel and Segal (2009). Such a limit argument would only show that the expected number of messages approaches infinity. In addition, we show that this strengthening is due to the specific payment rule of the Vickrey auction. For the case of 2 bidders with valuations from the unit interval, allowing for general Groves payments (Groves, 1973) somewhat mitigates the situation: though infinite running time does still occur with positive probability, it is no longer with probability one. We complete our analysis of the cost of selfishness in the case of continuous valuations by providing an analysis of the bisection protocol. Our analysis shows that, under obedient reporting with $n$ agents, efficiency can be achieved with finite running time almost always, and an average number of binary messages equal to $2 n$.

\section{Preliminaries}

A single indivisible object is being sold to one individual from a set $N=\{1, \ldots, n\}$ of bidders by means of a deterministic mechanism. The set of messages of bidder $i$ is denoted by $F_{i}$. A message profile is a vector $f=\left(f_{i}\right)_{i \in N} \in F$ of messages, where $F=\prod_{i \in N} F_{i}$. The winner-determination rule $w: F \rightarrow N$, decides for each message profile $f$, who wins the object. The payment function $p_{i}: F \rightarrow \mathbb{R}$ determines, for each message profile $f$, the amount $p_{i}(f)$ that bidder $i$ has to pay to the auctioneer. A payment profile is a vector $p=\left(p_{i}\right)_{i \in N}$ of payment functions. A triplet $(F, w, p)$ is called a mechanism. Each bidder has a valuation $v_{i}$ for the object. Valuations are drawn from a non-degenerate interval $I=[a, b]$ with $a \geq 0$, and are assumed to be private information. A valuation profile is a vector $v=\left(v_{i}\right)_{i \in N}$ of valuations. A strategy of bidder $i, s_{i}: I \rightarrow F_{i}$, is a function that states, for each valuation $v_{i}$, which message $s_{i}\left(v_{i}\right)$ the bidder will choose. A strategy profile is a vector $s=\left(s_{i}\right)_{i \in N}$ of strategies.

A realization of the valuation profile $v$ defines an ex post game $(F, w, p, v)$, with message space $F_{i}$ for bidder $i$ and payoff function $u_{i}\left(v_{i}\right): F \rightarrow \mathbb{R}$ given by

$u_{i}\left(v_{i}\right)(f):= \begin{cases}v_{i}-p_{i}(f) & \text { if } i=w(f) \\ -p_{i}(f) & \text { otherwise. }\end{cases}$

A message profile $f$ is a Nash equilibrium of the ex post game $(F, w, p, v)$ if, for every bidder $i$ and every message $g_{i} \in F_{i}$ of that bidder, it holds that

$u_{i}\left(v_{i}\right)(f) \geq u_{i}\left(v_{i}\right)\left(f \mid g_{i}\right)$ where $\left(f \mid g_{i}\right)$ denotes the message profile where bidder $i$ chooses $g_{i}$ and every other bidder $j$ chooses $f_{j}$. A strategy profile $s$ is an ex post equilibrium of the mechanism $(F, w, p)$ if, for every realization of the valuation profile $v$, the message profile $s(v):=\left(s_{i}\left(v_{i}\right)\right)_{i \in N} \in F$ is a Nash equilibrium of the ex post game $(F, w, p, v)$. A strategy profile $s$ is called ex post efficient if, for every realization $v$, it holds that

$w(s(v)) \in \arg \max \left\{v_{i} \mid i \in N\right\}$.

A mechanism $(F, w, p)$ is called direct if $F_{i}=I$ for each bidder $i$. In a direct mechanism it is clear what the message spaces are, so we write $(w, p)$ to denote such a mechanism. Note that a strategy profile $s$ in $(F, w, p)$ induces a direct mechanism ( $w \circ s, p \circ s)$. A direct mechanism $(w, p)$ is called a Vickrey-Clarke-Groves mechanism (a VCG mechanism) if, for every bidder $i$, there exists a function $h_{i}: I^{N \backslash\{i\}} \rightarrow \mathbb{R}$ such that, for every profile $r=\left(r_{i}\right)_{i \in N}$ of reported valuations in $I^{N}$, it holds that

$w(r) \in \arg \max \left\{r_{i} \mid i \in N\right\}$
$p_{i}(r)= \begin{cases}h_{i}\left(r_{-i}\right) & \text { and } \\ h_{i}\left(r_{-i}\right)-\max \left\{r_{j} \mid j \neq i\right\} & \text { otherwise. }\end{cases}$

The Vickrey mechanism is the VCG mechanism with $h_{i}\left(r_{-i}\right)=$ $\max \left\{r_{j} \mid j \neq i\right\}$.

DIGITAL IMPLEMENTATION. VCG mechanisms have many appealing properties, such as dominant strategy solvability, and ex post efficiency in equilibrium. Despite these theoretical advantages, VCG and Vickrey mechanisms are hardly ever used in their pure form to allocate goods. Usually these mechanisms are implemented via an iterative procedure so that information regarding bidders' valuations is gradually elicited. Such iterative procedures are referred to as digital mechanisms.

In a digital mechanism, the auctioneer sequentially offers bidders the opportunity to take one of a finite set of actions. Such an offer is referred to as a query. During the execution of the mechanism each bidder may be, and usually will be, queried more than once. Determination of the winner and the payments in the mechanism are based exclusively on the actions taken by the bidders in response to the queries of the auctioneer. The digital mechanism ends as soon as the winner and all payments have been determined. The number of times a particular bidder is queried during the mechanism is not assumed to be bounded. Consequently, a digital mechanism may potentially take an infinite number of query rounds.

Digital mechanisms are special cases of mechanisms as defined above, where the complete set of actions that a bidder plans to take in the extensive form game induced by the digital mechanism defines his message. Let thus $(F, w, p)$ be a digital mechanism, and let $s=\left(s_{i}\right)_{i \in N}$ be an ex post equilibrium in this mechanism. Then the digital mechanism $(F, w, p)$ implements a VCG choice rule (Vickrey choice rule) via $s$ if the direct mechanism $(w \circ s, p \circ s)$ is a VCG mechanism (Vickrey mechanism).

\section{The communication cost of selfishness}

Let $(F, w, p)$ be a digital mechanism, and let $s$ be an ex post equilibrium in the mechanism. Let $Z$ be the set of valuation profiles $v$ for which, in the message profile $s(v)$, the auctioneer asks a finite number of queries before the mechanism terminates. When the Lebesgue measure of the set $Z$ is equal to zero, the equilibrium $s$ is said to almost always have infinite running time.

Theorem 3.1. Suppose that mechanism $(F, w, p)$ implements the Vickrey choice rule via ex post equilibrium $s$. Then s almost always has infinite running time. 
Proof. Consider an ex post equilibrium $s$ under mechanism $(F, w, p)$. Suppose that $(w \circ s, p \circ s)$ is a Vickrey mechanism. We show that $Z$ has Lebesgue measure zero.

For $i \in N$ and $k \in \mathbb{N}$, let $Z(i, k)$ be the set of valuations $v$ such that $(w \circ s)(v)=i$ and the mechanism ends after exactly $k$ queries. Further, for $r \in \mathbb{R}$, let $Z(i, k, r)$ be the set of valuations $v \in Z(i, k)$ such that $(p \circ s)(v)=r$. Let $R(i, k)$ be the set of prices $r$ such that $Z(i, k, r)$ is not empty. Since the mechanism is deterministic, $R(i, k)$ is finite. Further, ( $w \circ s, p \circ s)$ is a Vickrey mechanism, so $(p \circ s)(v)=\max \left\{v_{j} \mid j \in N \backslash\{i\}\right\}$ for any $v$ and $i$ such that $(w \circ s)(v)=i$.

Thus, $\quad Z(i, k, r) \subseteq \bigcup_{j \in N \backslash\{i\}}\left\{v \in I^{n} \mid v_{j}=r\right\}$,

and $Z(i, k, r)$ is contained in a set with Lebesgue measure 0 . Hence, also

$Z=\bigcup_{i \in N} \bigcup_{k \in \mathbb{N}} \bigcup_{r \in R(i, k)} Z(i, k, r)$

has Lebesgue measure zero.

As a result, implementation of the Vickrey choice rule by means of a digital mechanism can only be achieved at the expense of a running time of the mechanism that is infinite for almost all realizations. Consequently, when valuations are drawn from a continuous probability distribution, the digital mechanism will necessarily run for an infinite amount of time in equilibrium with probability one.

Would the goal only be efficiency, and would bidders report obedient in a digital mechanism, then far less communication would be needed. Indeed, consider the following simple bisection search protocol. Initially there are $n$ bidders, each bidder $i$ having a valuation $v_{i} \in[a, b]$ randomly drawn from a uniform distribution. The auctioneer asks each bidder whether his valuation exceeds $q_{1}=\frac{a+b}{2}$. When all bidders report zero ("no"), all bidders enter the next round, and we continue our search protocol on the interval $\left[a, q_{1}\right)$. When precisely one bidder reports one ("yes"), this bidder gets the item, and the protocol terminates. When $k \geq 2$ bidders report one, all such bidders enter the next round, and the search protocol continues on the interval $\left[q_{1}, b\right]$. This process continues until exactly one bidder reports one.

Proposition 3.2. When $n \geq 2$ bidders report obediently, the bisection protocol has finite running time almost always. Moreover, the expected number of bits transmitted is $2 n$.

Proof. The bisection protocol has finite running time on any profile $\left(v_{i}\right)_{i \in N}$ of valuations for which $v_{i} \neq v_{j}$ for all $i \neq j$. Hence, the bisection protocol has finite running time almost always.

Consider the bisection protocol with $n$ obedient bidders. Let $E(k)$ denote the expected number of bits transmitted under the protocol starting with $k$ obedient bidders. Initially all $n$ bidders are queried, which results in $n$ communicated bits. Suppose that $k$ bidders report a one. When $k=1$, the protocol terminates. With probability $2 \cdot\left(\frac{1}{2}\right)^{n}=\left(\frac{1}{2}\right)^{n-1}$, we have $k=0$ or $k=n$, and the number of bidders for the next query round remains $n$. With probability $\left(\begin{array}{l}n \\ k\end{array}\right) \cdot\left(\frac{1}{2}\right)^{n}$, the protocol continues with $k$ bidders. Thus we obtain

$\left(1-\left(\frac{1}{2}\right)^{n-1}\right) \cdot E(n)=n+\sum_{k=2}^{n-1}\left(\begin{array}{l}n \\ k\end{array}\right) \cdot\left(\frac{1}{2}\right)^{n} \cdot E(k)$.

We show by induction that $E(n)=2 n$ for all $n$. For $n=2$ we obtain $\frac{1}{2} \cdot E(2)=2$, so that $E(2)=4$. Suppose that $E(k)=2 k$ for all $k<n$. Substitution into the above equation yields

$\left(1-\left(\frac{1}{2}\right)^{n-1}\right) \cdot E(n)=n+\left(\frac{1}{2}\right)^{n-1} \cdot\left[\sum_{k=2}^{n-1}\left(\begin{array}{l}n \\ k\end{array}\right) \cdot k\right]$.

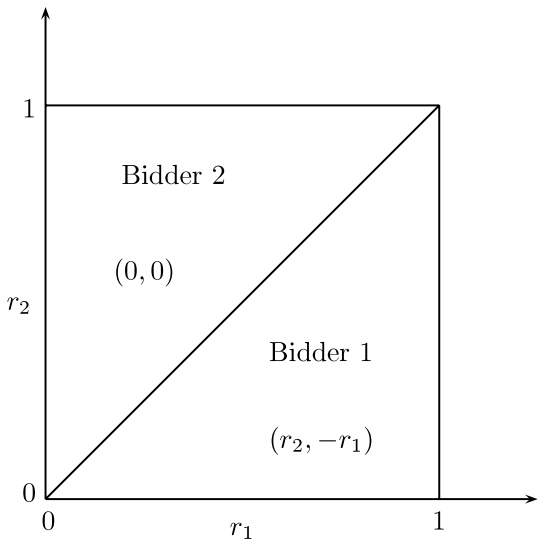

Fig. 1. The direct auction $\left(w^{*}, p^{*}\right)$.

Now notice that $\sum_{k=2}^{n-1}\left(\begin{array}{l}n \\ k\end{array}\right) \cdot k=n \cdot 2^{n-1}-2 n$. Substitution of this equality into the previous equality yields $E(n)=2 n$.

Search protocols with obedient reporting were also studied in Arrow et al. (1981). Their results mainly concern the number of queries needed to find the $k$ highest valuations. They find an upper bound of approximately 2.5 for the expected number of queries. We find $2 n$ for the expected number of bits transmitted. As in Arrow et al. (1981), the bisection protocol can be adjusted to any continuous distribution without changing the result.

Hence, when bidders report obediently, efficiency can be achieved under a protocol that has finite running time almost always, even with finite expected communication cost. ${ }^{4}$ Thus, the fact that implementation of the Vickrey choice rule requires almost always infinite running time is an overhead cost that is due to strategic effects. For a setting with finite sets of valuations, Fadel and Segal (2009) show that the extra communication needed to guarantee efficiency in ex post equilibrium goes to infinity if the number of valuations goes to infinity. Taking a limit argument does not rule out the existence of digital mechanisms in a setting with continuous valuations that have finite communication cost with strictly positive probability. The following example shows that such mechanisms indeed exist. It follows from Green and Laffont (1977) that such a digital mechanism necessarily implements a VCG mechanism. However, due to Theorem 3.1 the payment is not the Vickrey payment, but a general Groves payment.

EXAMPLE. Let $N=\{1,2\}$ and $I=[0,1]$. The following is an example of a mechanism $(F, w, p)$ and an ex post equilibrium $s$ in that protocol such that the direct auction $(w \circ s, p \circ s)$ equals the following direct auction $\left(w^{*}, p^{*}\right)$. Define $w^{*}$, based on reported valuations $\left(r_{1}, r_{2}\right)$, by $w^{*}\left(r_{1}, r_{2}\right)=1$ precisely when $r_{1} \geq r_{2}$. So, bidder 1 is always the winner in case of a tie. Define the payments $p^{*}=\left(p_{1}^{*}, p_{2}^{*}\right)$ by

$p_{1}^{*}\left(r_{1}, r_{2}\right)=\left\{\begin{array}{ll}r_{2} & \text { if } r_{1} \geq r_{2} \\ 0 & \text { else }\end{array} \quad\right.$ and $\quad p_{2}^{*}\left(r_{1}, r_{2}\right)= \begin{cases}-r_{1} & \text { if } r_{1} \geq r_{2} \\ 0 & \text { else. }\end{cases}$

So, bidder 1 is charged Vickrey prices. However, bidder 2 does not pay anything when he wins the object (in case $r_{2}>r_{1}$ ), and he receives an amount of $r_{1}$ (the report of the other bidder) in case he loses (when $r_{2} \leq r_{1}$ ). See the Fig. 1 for an illustration. This is in fact a VCG mechanism, where $h_{1}\left(r_{2}\right)=r_{2}$, and $h_{2}\left(r_{1}\right)=0$.

Since $\left(w^{*}, p^{*}\right)$ is a VCG choice rule, truthful reporting is a dominant strategy. The following mechanism implements the direct auction $\left(w^{*}, p^{*}\right)$ via truthful reporting. Notice that we have

\footnotetext{
4 Finite communication cost in expectation is not an automatic consequence of almost always finite running time.
} 
to determine whether either $r_{2}>r_{1}$, in which case the auction ends, or $r_{2} \leq r_{1}$, in which case full elicitation of valuations is needed to implement the payments.

We first give a detailed description of each round of the query auction. At the start of each round parameters $l_{k 1}, l_{k 2}, u_{k 1}, u_{k 2}$, and $w_{k-1}$ are given. For $i=1,2$, the numbers $l_{k i}$ and $u_{k i}$ are interpreted as the respective current lower and upper bound on the valuation $v_{i}$ of bidder $i$. The value of $w_{k-1}$ can be 0,1 , or 2 . If $w_{k-1}=0$ the winner has not been found yet. If $w_{k-1}=1$, the winner is bidder 1 . If $w_{k-1}=2$, the winner is bidder 2 .

START. We define $l_{11}=l_{12}=0, u_{11}=u_{12}=1$, and $w_{0}=0$.

ROUND $k$. Given are $l_{k 1}, l_{k 2}, u_{k 1}, u_{k 2}$, and $w_{k-1}$. Define $q_{k 1}=$ $\frac{l_{k 1}+u_{k 1}}{2}$, and $q_{k 2}=\frac{l_{k 2}+u_{k 2}}{2}$. Let each bidder respond to the query "Is your valuation $v_{i}$ larger than or equal to $q_{k i}$ ?". Both bidders are allowed to choose an element of the set $\{0,1\} .^{5}$ Reporting a zero is interpreted as a "no", while reporting one signifies "yes". This implicit interpretation gives meaning to the phrase "truthful reporting", and is also reflected in the allocation and payment schemes determined by the responses of the bidders.

If bidder 1 reports zero, bidder 2 reports one, and $w_{k-1}=0$. Set $w_{k}=2$ (bidder 2 wins), and the protocol terminates.

If bidder 1 reports zero, bidder 2 reports one, and $w_{k-1} \neq 0$. Set $w_{k}=w_{k-1}, l_{k+1,1}=l_{k 1}, u_{k+1,1}=q_{k 1}, l_{k+1,2}=q_{k 2}$, and $u_{k+1,2}$ $=u_{k 2}$. Go to round $k+1$.

If bidder 1 reports one, and bidder 2 reports zero. If $w_{k-1}=0$, set $w_{k}=1$. Else $w_{k}=w_{k-1}$. Further, $l_{k+1,1}=q_{k 1}, u_{k+1,1}=u_{k 1}$, $l_{k+1,2}=l_{k 2}$, and $u_{k+1,2}=q_{k 2}$. Go to round $k+1$.

If bidder 1 reports zero, and bidder 2 reports zero. Set $w_{k}=$ $w_{k-1}$. Further, $l_{k+1,1}=l_{k 1}, u_{k+1,1}=q_{k 1}, l_{k+1,2}=l_{k 2}$, and $u_{k+1,2}=$ $q_{k 2}$. Go to round $k+1$.

If bidder 1 reports one, and bidder 2 reports one. Set $w_{k}=w_{k-1}$. Further, $l_{k+1,1}=q_{k 1}, u_{k+1,1}=u_{k 1}, l_{k+1,2}=q_{k 2}$, and $u_{k+1,2}=u_{k 2}$. Go to round $k+1$.

FINISH. When the protocol took a finite number of rounds. In this case bidder 2 wins the object, and both bidders have zero payment.

When the protocol took an infinite number of rounds. In this case bidder 1 wins the object. For bidder 1 , the payment is $p_{1}=$ $\sup \left\{l_{k 2}\right\}$. Bidder 2 receives an amount of $p_{2}=\sup \left\{l_{k 1}\right\}$.

It is straightforward to check that, in this mechanism, truthful reporting is an ex post equilibrium. Moreover, the induced direct auction is exactly $\left(w^{*}, p^{*}\right)$. Notice that the mechanism terminates after a finite number of steps precisely when bidder 2 wins the auction. The description of the induced direct auction $\left(w^{*}, p^{*}\right)$ shows that this is the case when $v_{2}>v_{1}$. Hence, the Lebesgue measure of $Z$ equals $\frac{1}{2}$, and $Z$ does not have Lebesgue measure zero.

This is surprising in the sense that, when we only require to implement a VCG choice rule instead of the Vickrey choice rule, the mechanism may end in finite time with positive probability, while this is not possible when we insist on the Vickrey choice rule.

\section{Two bidders}

For two bidders, we can sharpen the result of Theorem 3.1 somewhat. In the general case with $n$ bidders, the requirement is that the ex post equilibrium $s$ allocates efficiently for any realization of valuations (that is, $s$ is ex post efficient). Then we can conclude that the running time is infinite for almost all realizations of valuations. For two bidders, it is only necessary to require that the ex post equilibrium $s$ allocates efficiently for a set of valuation pairs that has full Lebesgue measure to draw the same conclusion, namely that the running time must be infinite almost always.

Theorem 4.1. Let $(F, w, p)$ be a digital mechanism with two bidders that implements the Vickrey choice rule via ex post equilibrium $\mathrm{s}$. If the set of valuation pairs for which s does not allocate efficiently has Lebesgue measure zero, then s almost always has infinite running time.

Proof. Suppose that $s$ implements the Vickrey choice rule in $(F, w, p)$. If there is no pair of valuations for which $s$ allocates inefficiently, the result follows from Theorem 3.1. Suppose $\left(v_{1}, v_{2}\right)$ in $I \times I$ is a valuation pair for which $s$ allocates inefficiently. Suppose w.l.o.g. that $v_{1}>v_{2}$ and bidder 2 wins. Let $S$ be the set of pairs $\left(u_{1}, u_{2}\right)$ in $I \times I$ with $v_{2}<u_{1}<v_{1}$ and $v_{2}<u_{2}<v_{1}$ and $u_{2}<u_{1}$. We argue that bidder 2 also wins for any $\left(u_{1}, u_{2}\right) \in S$. Take $\left(u_{1}, u_{2}\right) \in S$. Because $s$ is an ex post equilibrium, $v_{2}<$ $u_{2}$, and bidder 2 wins at $\left(v_{1}, v_{2}\right)$, bidder 2 also wins at $\left(v_{1}, u_{2}\right)$. Analogously, bidder 2 also wins at $\left(u_{1}, u_{2}\right)$.

Thus, suppose that we want to construct a mechanism that allocates efficiently ex post, or even only efficiently ex post with probability one. Then we necessarily end up with a digital mechanism that in equilibrium runs an infinite amount of time with probability one.

\section{Acknowledgments}

The first author acknowledges support by the Dutch Science Foundation NWO through grant 401-01-101. The second author acknowledges support by the Dutch Science Foundation NWO through a VICI-grant. The authors thank Matt Embrey and an anonymous referee of their paper for very constructive comments that helped to improve the paper considerably.

\section{References}

Arrow, K.J., Pesotchinsky, L., Sobel, M., 1981. On partitioning a sample with binarytype questions in lieu of collecting observations. Journal of the American Statistical Association 76, 402-409.

David, E., Rogers, A., Jennings, N.R., Schiff, J., Kraus, S., Rothkopf, M.H., 2005. Optimal design of English auctions with discrete bid levels. ACM Transactions on Internet Technology 7 (2), 2-34.

Fadel, R., Segal, I., 2009. The communication cost of selfishness. Journal of Economic Theory 1895-1920.

Green, J., Laffont, J.J., 1977. Characterization of satisfactory mechanisms for the revelation of preferences for public goods. Econometrica 45, 427-435.

Grigorieva, E., Herings, P.J.J., Müller, R., Vermeulen, D., 2009. Fraction auctions: the tradeoff between efficiency and running time. METEOR research memorandum RM09045.

Groves, T., 1973. Incentives in teams. Econometrica 617-631.

Rothkopf, M.H., Harstad, R.M., 1994. On the role of discrete bid levels in oral auctions. European Journal of Operational Research 74, 572-581.

Vickrey, W., 1961. Counterspeculation, auctions and competitive sealed tenders. Journal of Finance 16, 8-37.

\footnotetext{
5 If one wants to have a proper iterative protocol, in which only one bidder reports per round, one can think of a round as two consecutive rounds, during which no extra information is communicated to the bidders.
} 